\title{
A Resource for Quantum computation
}

Niklas Johansson

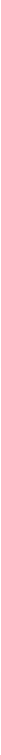



Linköping Studies in Science and Technology

Dissertation No. 2191

\title{
A Resource for Quantum Computation
}

Niklas Johansson

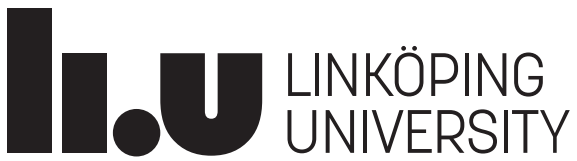

\author{
Information Coding Group \\ Department of Electrical Engineering \\ Linköping University \\ SE-581 83 Linköping, Sweden
}

Linköping 2021 
This is a Swedish Doctor of Philosophy thesis The Doctor of Philosophy degree comprises 240 ECTS credits of postgraduate studies.

Cover art: Undecided dices by Niklas Johansson

Copyright $\odot$ Niklas Johansson unless otherwise stated ISBN: 978-91-7929-123-5 (Print) ISBN: 978-91-7929-124-2 (PDF) ISSN 0345-7524

https://doi.org/10.3384/9789179291242

Printed by LiU-Tryck, Linköping, Sweden 2021 




\section{Abstract}

In this thesis we address the question, what is the resource, or property, that enables the advantage of quantum computers? The theory of quantum computers dates back to the eighties, so one would think there already is an answer to this question. There are several proposed solutions, but to this date, there is no consensus on an answer.

Primarily, the advantage of quantum computers is characterized by a speedup for certain computational problems. This speedup is measured by comparing quantum algorithms with the bestknown classical algorithms. For some algorithms we assume access to an object called oracle. The oracle computes a function, and the complexity of the oracle is of no concern. Instead, we count the number of queries to the oracle needed to solve the problem. Informally, the question we ask using an oracle is: if we can compute this function efficiently, what else could we then compute. However, using oracles while measuring a quantum speedup, we assume access to vastly different oracles residing in different models of computation.

For our investigation of the speedup, we introduce a classical simulation framework that imitates quantum algorithms. The simulation suggests that the property enabling the potential quantum speedup is the ability to store, process, and retrieve information in an additional degree of freedom. We then theoretically verified that this is true for all problems that can be efficiently solved with a quantum computer.

In parallel to this, we also see that quantum oracles sharply specify the information we can retrieve from the additional degree of freedom, while regular oracles do not. A regular oracle does not even allow for an extra degree of freedom. We conclude that comparing quantum with classical oracle query complexity bounds does not provide conclusive evidence for a quantum advantage. 



\section{Sammanfattning}

I denna avhandling behandlar vi frågan, vad är resursen eller egenskapen som möjliggör fördelen hos kvantdatorer. Teorin bakom kvantdatorer daterar tillbaka till åttiotalet, så man skulle kunna tro att det redan finns ett svar på frågan. Det finns flera föreslagna lösningar, men hittills råder det ingen enighet kring ett svar.

Fördelen hos kvantdatorer kännetecknas främst av en uppsnabbning för vissa beräkningsproblem. Denna uppsnabbning mäts genom att man jämför kvantalgoritmer med de bästa klassiska algoritmerna som vi känner till. För vissa algoritmer så antas att man har tillgång till ett objekt som kallas orakel. Ett orakel beräknar en funktion och vi bryr oss inte om komplexiteten hos oraklet. Istället så räknar vi antalet annrop man behöver göra till oraklet för att lösa problemet. Informellt så kan man säga att när man använder ett orakel så ställer vi frågan: om vi kan beräkna den här funktionen effektivt, vad kan vi då beräkna? Men när man använder orakel för att mäta en kvantuppsnabbning så antar vi att man har tillgång till väldigt olika orakel i olika beräkningsmodeller.

För vår undersökning av kvantuppsnabbningen introducerar vi ett klassiskt simuleringsramverk som imiterar kvantalgoritmer. Simuleringen antyder att egenskapen som möjliggör den potentiella kvantuppsnabbningen är förmågan att lagra, bearbeta och hämta information i en extra frihetsgrad. Vi verifierar sedan teoretiskt att detta är sant för alla problem som effektivt kan lösas med en kvantdator.

Parallellt med detta ser vi att kvantorakel entydigt specificerar den information vi kan hämta ur den extra frihetsgraden, medan vanliga orakel inte gör det. Ett vanligt orakel tillăter inte ens en extra frihetsgrad. Från detta drar vi slutsatsen att jämförelser mellan kvantmekanisk och klassisk anropskomplexitet inte kan ge övertygande bevis för en kvantfördel. 




\section{Acknowledgements}

I want to thank Jan-Åke Larsson, without whom this research would not have been possible. If there is any magic in the world, it shows through Jan-Åke's ability to simultaneously be a good supervisor, colleague, and friend.

I would also like to thank my co-supervisor Peter Jonsson, for many valuable discussions - not only restricted to theoretical computer science.

To my friends and collogues that have challenged me to become better, especially Jonathan Jogenfors. Thank you for your continued support.

And last but not least, I would like to thank my beloved family. To Egle for her love and encouragement, and for helping out in all the ways she could think of. To William, whose smile gave me strength to carry on writing, and Isabella for constantly reminding me of the importance of taking a play break. To my brothers, Fredrik and Christian, and their families for reminding me that there are other things to life than work. And to my parents Gunnar and Gunilla, one could not wish for better parents, and my highest goal in life is to be an equally good parent to William. 


\section{Contents}

1 Introduction 1

1.1 Measuring the Speedup . . . . . . . . . . . 2

1.2 Motivation ................ 4

1.3 Organization of the Thesis $\ldots \ldots \ldots 5$

$\begin{array}{lll}\text { I Preliminaries } & 7\end{array}$

2 Reversible Computation 9

2.1 Setting the Scene . . . . . . . . . . . 9

2.2 The Garbage of History . . . . . . . . . . . . . 11

2.3 Uncomputation . . . . . . . . . . . . . 13

2.4 Notation and Gates . . . . . . . . . . . 14

3 Quantum Computation 17

3.1 States and Transformations . . . . . . . . 17

3.2 Measurement ......................... 22

3.3 Gates and Notation ............ 24

II Summary of the results $\quad 29$

4 Results $\quad 31$

4.0.1 Publication A . . . . . . . . . . . . 31

4.0 .2 Publication B ........... 33 
4.0.3 Publication C . . . . . . . . . 34

4.0 .4 Publication D . . . . . . . . 36

4.1 Conclusions . . . . . . . . . . . 37

4.2 Future Work . . . . . . . . . . . 40

III Publications

A Efficient Classical Simulation of the Deutsch-Jozsa and Simon's Algorithms

A.1 Introduction . . . . . . . . . . . . 52

A.2 The Deutsch-Jozsa problem and quantum algorithm .................. 54

A.3 Quantum Simulation Logic . . . . . . . . . 57

A.4 Simulation of the Deutsch-Jozsa algorithm... . . . 60

A.5 Equal requirements on quantum and QSL oracles 63

A.6 Simon's problem ....................... 66

A.7 Conclusions . . . . . . . . . . 71

B Quantum Simulation Logic, Oracles, and the Quantum Advantage $\quad 77$

B.1 Introduction . . . . . . . . . . . 78

B.2 Preliminaries . . . . . . . . . . 82

B.2.1 Turing Machines . . . . . . . . . 82

B.2.2 Oracle Turing Machines and oracle notions 84

B.2.3 Quantum Computation ....... . 88

B.3 Quantum Simulation Logic . . . . . . . . . 94

B.3.1 Elementary Systems . . . . . . . . . 95

States . . . . . . . . . . . . 95

Transformations . . . . . . . . . 100

No Universal Spin-1/2 Inverter . . . . . . . 105

Measurement . . . . . . . . 106

Preparation .............. 107

Non-commutativity of Measurements . . 107

QKD - BB84 . . . . . . . . . . 108

B.3.2 Pairs of Elementary Systems . . . . . . . . 111 
Transformations . . . . . . . . . 111

Entanglement . . . . . . . . . 113

Remote Steering . . . . . . . . . 115

Anti-correlation in Spin-measurements of

the Singlet . . . . . . . 117

No-cloning . . . . . . . . . . . 118

Interference . . . . . . . . . 120

Measurements . . . . . . . . . . 124

Superdense Coding . . . . . . . . . 126

B.3.3 Higher Number of Elementary Systems . 127

Teleportation .................. 128

Transformations . . . . . . . . . 130

B.3.4 Properties and Relations to other Theories 131 The relation to stabilizer quantum mechan-

ics, locality and contextuality . . 131

QSL extends the state space of Spekkens'

model ............ 134

QSL is an example of a Generalized Prob-

ability Theory . . . . . . . 136

B.4 The Bernstein-Vazirani Problem . . . . . . . 139

B.4.1 Problem Formulation . . . . . . . . . . . 140

B.4.2 Classical Algorithm . . . . . . . . . . . 140

B.4.3 Quantum Algorithm ......... 140

B.4.4 QSL Simulation . . . . . . . . . . . . . 142

B.5 The Deutsch-JozsA Problem . . . . . . . . . . 144

B.5.1 Problem Formulation . . . . . . . . . . . 145

B.5.2 Deterministic and Probabilistic Algorithms146

B.5.3 Quantum Algorithm . . . . . . . . . . 147

B.5.4 The Problem for Small Input . . . . . . 148

B.5.5 QSL Simulation Guaranteed a Constant or Balanced Function . . . . . . . . . 150

B.5.6 QSL Simulation Accepting Arbitrary Boolean

Functions . . . . . . . . . . 154

B.5.7 Query complexity .......... 158

B.6 Oracles as a Comparison . . . . . . . . . . 159

B.6.1 The Additional Structure and Constraints 159 
B.6.2 Is the Black-Box Black? . . . . . . . . . . 161

B.6.3 Assumptions in the Use of Oracles . . . . 162

B.6.4 Systematic Phase Errors . . . . . . . . . 163

B.6.5 Starting with Something Else Than Access

to an Oracle . . . . . . . . . . 168

B.7 Grover's Algorithm . . . . . . . . . . . . 169

B.7.1 Problem Formulation . . . . . . . . . . 171

B.7.2 One-Shot Grover . . . . . . . . . . . . . 171

B.7.3 The $n$-Toffoli . . . . . . . . . . . . . 173

B.7.4 A Scaling Algorithm . . . . . . . . . 177

B.7.5 Comparison with a 3-qubit Experiment . 180

B.7.6 Application to Ciphers . . . . . . . . . . 181

B.8 Simon's Algorithm . . . . . . . . . . . . . 182

B.8.1 Problem Formulation . . . . . . . . . . 183

B.8.2 Probabilistic Solution . . . . . . . . . . . 183

B.8.3 Quantum Algorithm . . . . . . . . . 184

B.8.4 QSL Simulation . . . . . . . . . . . 187

B.8.5 Adding the function output to the target modulo $2 \ldots \ldots \ldots$. . . . . . 191

B.8.6 A deterministic algorithm for Simon's problem . . . . . . . . . . . 192

B.8.7 Application to Symmetric Ciphers . . . . 195

B.9 Shor's Algorithm Factoring 15 . . . . . . . . 197

B.10 Conclusion . . . . . . . . . . . . . 205

B.11 Constant and Balanced Functions for Three Bits of Input . . . . . . . . . . . . . . . . 216

B.12 Error Probability for Different Constructions of the Majority Function . . . . . . . . . . . 220

C Quantum Computation and the Additional Degrees of Freedom

C.1 Introduction . . . . . . . . . . . . . 224

C.2 Uncomputing garbage in reversible computation 227

C.3 Uncomputing garbage in quantum computation 230

C.4 The role of an extra degree of freedom in a BQPcomplete problem . . . . . . . . . . 237 
C.5 Circuit realization of the Hadamard test in the extra degree of freedom . . . . . . . . . . 240

C.6 Preserving relative phases through Bennett's trick 244

C.7 Developing quantum algorithms by finding the right degree of freedom . . . . . . . . . . 246

C.8 Optimizing quantum algorithms by focusing on the right degree of freedom . . . . . . . . . . . 249

C.9 Conclusion and Discussion . . . . . . . . . . 253

D Conjugate Logic $\quad 261$

D.1 Introduction . . . . . . . . . . . . . . . . 262

D.2 Conjugate logic propositions, negation, conjunction and disjunction . . . . . . . . . . . 265

D.3 Conjugate logic material and logical conditionals, tautology and contradiction . . . . . . . . 267

D.4 Equivalence relations and implication laws . . . 270

D.5 Fundamental uncertainty . . . . . . . . . . . 272

D.6 Transformations of propositions . . . . . . . . . 274

D.7 Composite system propositions and transformations 275

D.8 Clifford reduction . . . . . . . . . . . . . 278

D.9 Predictions . . . . . . . . . . . . . . . . 280

D.10 Measurements affect propositions . . . . . . . 281

D.11 Contextuality, and noncontextuality . . . . . . . 282

D.12 Conclusions . . . . . . . . . . . . . . . . 284

D.13 Truth tables for the logic equivalences and logic implications . . . . . . . . . . . . 290

D.14 Choice of $C Z$ transformation . . . . . . . . 298 



\section{Chapter 1}

\section{Introduction}

Landauer (1961) asked whether computation could be reversible and described with the dynamics of a closed physical system. He showed that the erasure of one bit of information must generate a corresponding amount of entropy, and in most computing machinery, this entropy is dissipated as heat. Erasure is an irreversible operation and cannot be allowed in a computational model aiming to be reversible. For a computation to be logically reversible, the input needs to be uniquely defined by the output: by knowing the output and the operations performed, we can reverse the computation to determine the input. A computation can always be made reversible by remembering the history of the computation. But as Landauer pointed out, any useful computer would eventually need to erase that history to reuse the memory it occupies. Bennett (1973) solved this problem by what we here refer to as Bennett's trick: first, perform the computation reversibly, then copy out the result to an additional register, and last, reverse the computation. In the last step, the history of the computation is uncomputed so that we can reuse the memory it occupied.

This research, about the physics of computation, also attracted 
the interest of Richard Feynman. He pointed out that there is no known tractable algorithm for simulating quantum theory, but if computers were built from quantum systems, they would most likely be able to (Feynman 1982).

So, if quantum computers can efficiently simulate quantum theory, but regular classical computers cannot, can quantum computers solve other computational problems that classical computers find intractable? The first indication of a positive answer came from Deutsch (1985) when he challenged the ChurchTuring hypothesis.

Every 'function which would naturally be regarded as computable' can be computed by the universal Turing machine.

- Alan Turing, as quoted by David Deutsch

Deutsch argues that there is a physical assertion underlying the Church-Turing hypothesis. He proposed that "functions which would naturally be regarded as computable" should be interpreted as "functions that are physically computable" . Deutsch then formalized the idea of a quantum Turing machine ${ }^{2}$ and exemplified how this machine can operate in a fundamentally different way than a regular Turing machine. This result is regarded by many as the starting point of quantum algorithm research

\subsection{Measuring the Speedup}

The computational power of quantum computers is mainly measured in terms of speed, that is, the number of operations needed to solve a computational problem (the problem's computational complexity). The complexity of a problem is specified by the

\footnotetext{
${ }^{1}$ The word "computable" here should not be confused with its use in computability theory. Instead, it should read as "tractable".

${ }^{2}$ A definition later strengthened by Bernstein and Vazirani (1993).
} 
currently best known algorithm for that problem, and if there is a quantum algorithm with lower complexity than the best known classical algorithm, the difference is referred to as the quantum advantage.

There are different ways to characterize the computational complexity of a problem. A commonly used method in quantum computation, that is of particular interest in this thesis, is the model called oracle query complexity.

In classical query complexity, we only get access to the input and output of a function and count the number of times we need to query it to solve a decision problem. In quantum query complexity, we instead get access to a unitary transformation that encodes the function. If the number of queries to the quantum oracle has a lower bound, and if this bound is lower than that for the classical query model, one considers this as evidence for a quantum advantage.

In query complexity, we count the number of queries to the oracle, and there is no promise that the function we get access to can be evaluated in polynomial time. Thus, a polynomially scaling bound does not imply a polynomial-time algorithm. Query complexity results are always relative to the oracle that one assumes access to, and the question asked is "if we could compute this function efficiently, what else could we then compute?".

In general, decision problems are categorized into complexity classes based on how resources scale with increasing problem size. A few examples follow. The complexity class $\mathbf{P}$ is the class of decision problems that can be solved deterministically in polynomial time. BPP is the class of decision problems that can be solved in polynomial time with an error probability bounded away from $1 / 2$. And $\mathbf{B Q P}$ contains all decision problems that a quantum computer can solve with a bounded error probability in polynomial time.

If there is a problem that is in $\mathbf{B Q P}$ but not in $\mathbf{P}$ (or $\mathbf{B P P}$ ), then 
we say that there is a separation between the two, i.e., there is a superpolynomial scaling of the gap between the complexity of the best known classical algorithm and the complexity of the best known quantum algorithm. Such problems are considered evidence for a quantum advantage.

\subsection{Motivation}

An open problem in quantum information processing is to understand what resources or properties that enable the speedup from quantum algorithms. Some properties that have been proposed as resources are superposition and interference (Feynman 1982; Bennett and DiVincenzo 2000), entanglement (Einstein, Podolsky, and Rosen 1935; Jozsa and Linden 2003; Briegel et al. 2009), nonlocality (Bell 1964; Bravyi, Gosset, and König 2018), contextuality (Kochen and Specker 1967; Larsson 2012; Howard et al. 2014; Bermejo-Vega et al. 2017), continuity of the state space (Shor 1994), the exponential growth of the state space (Arute et al. 2019), negative quasi-probability (Veitch et al. 2012; Bermejo-Vega et al. 2017), and even coherence (Baumgratz, Cramer, and Plenio 2014; Hillery 2016).

The goal of studying resources is to understand their relation to the advantage of quantum computation. Besides satisfying the academic curiosity, one could also hope that this understanding would contribute to the development of the field. For instance, if a resource could be ascribed as enabling the computational speedup, a hardware implementation could focus on this resource and hopefully get good results. Also, knowing what enables the speedup, it might be easier to find useful quantum algorithms that can exploit this resource.

Usually, resources are studied by starting with a quantum computer that is restricted to the stabilizer subtheroy of quantum mechanics, that is, we are limited to use Clifford group transformations, Pauli measurements, and classical controls. This 
subtheory does not allow for universal quantum computation. In fact, there is an efficient classical simulation for this part of quantum theory (Gottesman 1998; Aaronson and Gottesman 2004). Such a restricted quantum computer can be promoted to a universal one by adding resource states to the subtheory. Howard et al. (2014) showed that contextuality plays a critical role for finding these resource states, and conclude that contextuality ${ }^{3}$ provides the speedup.

Larsson 2012 worked on a model (Spekkens 2007) similar to the stabilizer subtheory, but different in that it does not contain quantum phenomena such as non-local and contextual correlations. Working on adding contextual relations, Larsson saw that the classical resources grow faster than the number of quantum systems. We thought that if we could modify this model to simulate quantum algorithms, we could better understand of how contextuality provides the speedup. That is, instead of observing the speedup of the quantum algorithm, we would observe the slowdown of its corresponding classical simulation, as it needed to account for more and more contextual correlations. We needed a specific quantum algorithm to work with, and decided on the Deutsch-Jozsa algorithm (Deutsch and Jozsa 1992). It did not go as we had hoped, but the research that followed from pursuing this idea is the subject of this thesis.

\subsection{Organization of the Thesis}

In Part I, we briefly go through some preliminaries that will be useful for the rest of the thesis. It should not be seen as a complete account of all different subjects, but rather as a refreshment of the most essential parts. In Part II, we will summarize the results from the four publications included in Part III, draw

\footnotetext{
${ }^{3}$ Contextuality here is called state-dependent and differs from the regular state-independent contextuality. After all, the stabilizer subtheory does contain the regular concept of contextual relations.
} 
some conclusions and point towards possible further work. The publications included are:

Pub. A. Niklas Johansson and Jan-Åke Larsson (2017). "Efficient Classical Simulation of the Deutsch-Jozsa and Simon's Algorithms”. In: Quantum Inf Process 16, p. 233. DOI: $10.1007 / \mathrm{s} 11128-017-1679-7$

Pub. B. Niklas Johansson and Jan-Åke Larsson (2019). "Quantum Simulation Logic, Oracles, and the Quantum Advantage". In: Entropy 21, p. 800.

DOI: $10.3390 / \mathrm{e} 21080800$

Pub. C. Niklas Johansson and Jan-Åke Larsson (2021). "Quantum computation and the additional degrees of freedom". In: Preprint

Pub. D. Niklas Johansson, Felix Huber, and Jan-Åke Larsson (To appear). "Conjugate Logic”. In: The Quantum-Like Revolution - A Festschrift for Andrei Khrennikov. Ed. by A. Plotnitsky and E. Haven. Springer 


\section{Part I}

\section{Preliminaries}





\section{Chapter 2}

\section{Reversible Computation}

This chapter will briefly review reversible logic, which is of utmost importance for the rest of the thesis. We usually think of it as the closest classical analog to quantum computing, and it is essential for understanding the circuit model.

Classical reversible logic is also a central part of the logical framework that we introduce in Publication A and B.

\subsection{Setting the Scene}

A machine performing a computation produces an output by acting on its input according to a given set of rules. Informally, a deterministic machine is a machine where for each possible state (or configuration), the next state is uniquely defined. For non-deterministic machines, this is not necessarily true; for a given state, the next one could be a set of many possible states. A special kind of non-deterministic machine is the probabilistic machine, where a probability distribution over the next-set-ofstates describes a transition from one state into the next. This kind of machine can be simulated by deterministic machines 
with additional external inputs providing randomness (Arora and Barak 2009, p. 124).

For a closed physical system, the evolution laws are reversible, and it is not hard to imagine that we could describe a deterministic machine in a closed physical system. For each system evolving from an initial state to a final state, one can set up a system evolving from the final state to the initial state. However, mathematical models of computation are rarely reversible. As an example, take the NAND gate (logical conjunction followed by negation). With $\boldsymbol{a}$ and $\boldsymbol{b}$ as bits, the output of this gate can be written as $\boldsymbol{a} \wedge \boldsymbol{b} \oplus 1$, where $\oplus$ represents addition modulo 2 , and $\wedge$ the logical conjunction. This gate merges two bits into one,

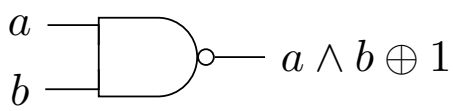

Figure 2.1: A NAND gate mapping $a$ and $b$ to $a \wedge b \oplus 1$.

which is a logically irreversible operation. Knowing the output to $a \wedge b \oplus 1$, we cannot go back and determine the initial states of $\boldsymbol{a}$ and $\boldsymbol{b}$.

In 1961, Landauer studied the relation between logical and physical reversibility. He proposed that logically irreversible operations, carried out by a machine, must be followed by an entropy increase in the machine's non-information-carrying degrees-offreedom. Typically, an irreversible computation dissipates heat to its environment.

He defined logical reversibility as the following.

Definition 2.1.1: A function is called logically reversible if the output uniquely defines the input.

Asserting that all inputs are well defined, it follows that the 
function is a bijection. If the function is a mapping from bitvectors to bit-vectors, there will be an equal amount of input bits as output bits since the domain and range must be of equal size.

\subsection{The Garbage of History}

To implement a function $f:\{0,1\}^{n} \rightarrow\{0,1\}^{m}$ reversibly, two registers are used. One input-register where we write the input $x$, and one output-register where we write the answer $f(x)$. The output of the input-register is called garbage, storing the history of the computation so that it can be reversed. The input to the output-register is called bias. It is commonly initiated with all bits cleared to enable writing the answer directly to this register. (see Figure 2.2.)

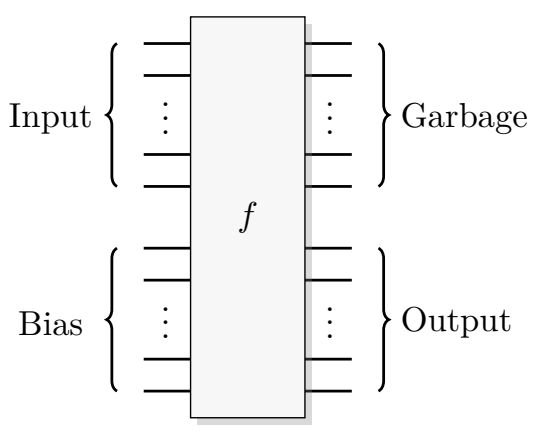

Figure 2.2: Reversible function showing the input- and outputregister with their bits as horizontal lines. It also shows the usual name conventions of the inputs and outputs to these registers.

Example 2.2.1: Consider turning the NAND gate in Figure 2.1 into a reversible gate. The gate performs a 2-to- 1 map, two inputs and one output. Let us call the inputs $\boldsymbol{a}$ and $\boldsymbol{b}$, and we will also need a 1-bit output-register. For simplicity, we choose the bias input $s=1$. On the output side, we need two garbage bits $g_{0}, g_{1}$ and one function output $a \wedge b \oplus 1$. We can choose the 
garbage bits to be equal to the inputs, i.e., $g_{0}=a$ and $g_{1}=b$. If we now add $\boldsymbol{a} \wedge \boldsymbol{b}(\bmod 2)$ to the output-register, and we are done. The truth table can be seen in Table 2.1, and we can verify that the information available on the output side is sufficient to determine what was given as input.

\begin{tabular}{ccc|ccc}
$a$ & $b$ & $s$ & $g_{0}$ & $g_{1}$ & $a \wedge b \oplus s$ \\
\hline 0 & 0 & 1 & 0 & 0 & 1 \\
0 & 1 & 1 & 0 & 1 & 1 \\
1 & 0 & 1 & 1 & 0 & 1 \\
1 & 1 & 1 & 1 & 1 & 0
\end{tabular}

Table 2.1: Truth table for NAND converted into a reversible function.

In general, we can represent these two registers as a tuple, $(\boldsymbol{x}, \boldsymbol{y})$, where $\boldsymbol{x}$ is the input-register and $\boldsymbol{y}$ the output-register. The reversible map $\boldsymbol{R}_{f}$ encoding $f$ we can define as

$$
R_{f}(x, 0)=\left(g_{R_{f}}(x), f(x)\right),
$$

where we choose to initiate the bias with all bits cleared. Observe that the garbage, $\boldsymbol{g}_{\boldsymbol{R}_{f}}(\boldsymbol{x})$, depends on the input $\boldsymbol{x}$ and on how $\boldsymbol{R}_{f}$ is implemented. To see why, consider the reversible implementation of NAND in Example 2.2.1. There we choose the garbage to be the same as the input, but we could have chosen the garbage differently. For instance, it could be swapped in place with $g_{0}=b \neq a$ and $g_{1}=a \neq b$. That would still be a reversible implementation, and the function $f$ identical, but the garbage is different.

A computation, in general, is not only evaluating a single function, but usually a sequence of many consecutive calculations. A computation is said to be reversible if all its intermediate steps are reversible.

Landauer (1961) pointed out two difficulties with reversible computation. The first difficulty is regarding bias. Consider 
a reversible machine aimed at evaluating a truth function constructed from NAND operations. To directly implement this with the reversible NAND operation described in Table 2.1, one would initiate the same amount of bias bits set to 1 as the number of NAND operations. This implementation would lead to an undesirable scaling of memory usage.

The second difficulty is that of having to store garbage. For example, Consider a computation containing many consecutive calculations. Since all intermediate steps need to be reversible, it is reasonable to assume that the history of each step needs to be stored. This would also lead to an undesirable scaling of memory usage.

Further, most programs are not self-terminating, they run indefinitely, and in this case, allocating memory for bias and garbage is clearly not feasible.

\subsection{Uncomputation}

Bennett (1973) showed a way to overcome these difficulties by reusing the memory allocated for bias and garbage. His method is known as Bennett's trick of uncomputation, and the idea is the following. Say that we want to reversibly compute a function $\boldsymbol{f}(\boldsymbol{x})$ from $\boldsymbol{n}$ to $\boldsymbol{m}$ bits, where $\boldsymbol{m} \leq \boldsymbol{n}$. As usual, we initiate an input-register of $\boldsymbol{n}$ bits in the state $\boldsymbol{x}$, and an output-register of $\boldsymbol{m}$ bits with the bias cleared. Additionally, we also initiate a blank auxiliary-register of $\boldsymbol{m}$ bits, aimed at assisting in the computation. The function is computed and written to the auxiliaryregister

$$
(x, 0,0) \mapsto\left(g_{R_{f}}(x), f(x), 0\right) .
$$

Next, the auxiliary-register is copied to the output-register,

$$
\left(g_{R_{f}}(x), f(x), 0\right) \mapsto\left(g_{R_{f}}(x), f(x), f(x)\right) .
$$

Observe that copying information to a register can be done reversibly without generating any garbage (the output uniquely 
defines the input). As the last step, the map in (2.2) is reversed

$$
\left(g_{R_{f}}(x), f(x), f(x)\right) \mapsto(x, 0, f(x)) .
$$

Overall we have

$$
(x, 0,0) \mapsto(x, 0, f(x)) .
$$

A gate representation of this procedure can be seen in Figure 2.3.

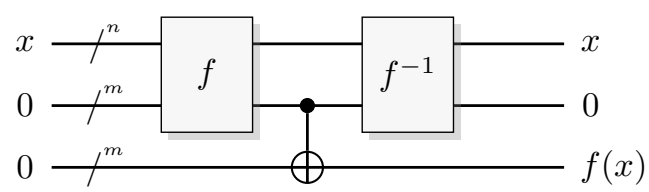

Figure 2.3: Gate representation of Bennett's trick of uncomputation. The middle operation represents a way of reversibly copy the information from the middle auxiliary-register to the lower output-register.

Note that the auxiliary-register, used for bias, is cleared and can be reused later in the computation. Also, that this procedure only produces a constant overhead in resources (time and space).

\subsection{Notation and Gates}

A powerful tool for reversible logic is the circuit representation. In this section, we will describe the circuit representation and the most commonly used gates.

As shown above, we represent bits in the circuit by horizontal lines, and the computational steps proceed from left to right.

The only single bit gate is the NOT gate which flips the bit value of the input, see Figure 2.4.

For two-bit gates, the most common is the controlled-NOT (CNOT) gate, which flips one bit if the other one is set. In other words, 


\section{$a \multimap a \oplus 1$}

Figure 2.4: Gate representation of the NOT gate, inverting the bit value of the input.

it adds one of the bits to the other one modulo 2. Similar to how the NOT gate relates to the regular inverter gate, the CNOT relates to the regular exclusive-OR gate. See Figure 2.5. The

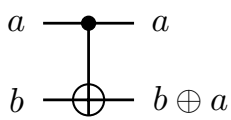

Figure 2.5: Gate representation of the CNOT gate, adding $\boldsymbol{a}$ to $\boldsymbol{b}(\bmod 2)$ and storing the result in the target.

upper bit $\boldsymbol{a}$ is usually called control, and the lower bit $\boldsymbol{b}$ is called target. This gate is sometimes called Feynman gate, referring to (Feynman 1985), but appeared already in (Toffoli 1980) under the name Xor/FAN-OUT.

Another useful two-bit gate is the SWAP (Feynman 1985), and it does precisely that, it swaps the input of two bits. See Figure 2.6 for the gate representation and its construction from CNOT gates.

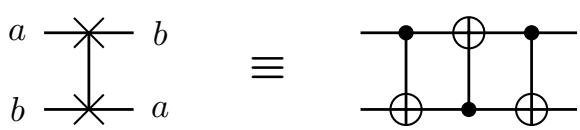

Figure 2.6: Gate representation of the SWAP gate and its construction from CNOT gates.

Next is the Toffoli gate that flips the target bit if both control bits are set. See Figure 2.7A. This gate appeared in (Landauer 1961), but now bears the name of Toffoli (1980), who generalized the 

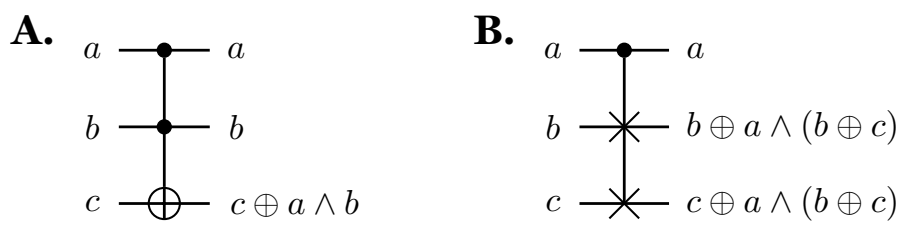

Figure 2.7: Gate representation of A. the Toffoli gate, flipping the target bit if the two controls are set $\mathbf{B}$. Fredkin gate. If $\boldsymbol{a}=\mathbf{0}$, then the output is the same as the input. And if $a=1$, then $b$ and $c$ are swapped.

gate to handle an arbitrary number of controls. Such a gate, with $n$ control bits, is referred to as an $\boldsymbol{n}$-Toffoli.

The last gate that we introduce here is called Fredkin gate, a controlled SWAP (Fredkin and Toffoli 1982), see Figure 2.7B. The Toffoli gate and the Fredkin gate are both universal for classical computation. 


\section{Chapter 3}

\section{Quantum Computation}

In quantum information theory, the units of information are the same as in classical information theory, but the information carriers are different. Their behavior is described by the statistical model called quantum theory, which is incompatible with the regular statistics used in classical information theory. This motivates the introduction of a new information theory.

Qubit is the name of the most elementary system described by quantum theory. It is not a two-state system as a bit, instead, the state lives in a two-dimensional projective Hilbert space. Measuring a qubit, however, we can only learn 1 bit of information. For us, a quantum computer will be a machine that does information processing on qubits. Certainly, there are information carriers with higher-dimensional Hilbert spaces, but for our purpose, qubits will suffice. Most of what is covered in this section can be found in greater detail in Nielsen and Chuang (2010).

\subsection{States and Transformations}

A quantum system is described by a quantum state $|\psi\rangle$ that we can represent by a complex vector in a closed inner product space, 
a Hilbert space. More specifically, the state space in quantum theory is a projective Hilbert space, where the complex vectors $|\psi\rangle$ and $e^{i \phi}|\psi\rangle$ are indistinguishable and represented by a single ray. The constant $e^{i \phi}$ is called the system's global phase and is not an observable property. We will, however, keep to the formulation of quantum states as complex vectors and just have to remember that global phases cannot be observed. We will also restrict ourselves to a finite-dimensional state space.

The notation for complex vectors used above is called Dirac's bra-ket notation, where $|\psi\rangle$ is called a ket and its dual $\langle\psi|$ is called bra,

$$
\langle\psi|=| \psi\rangle^{\dagger},
$$

where $(\dagger)$ denotes the hermitian conjugate (transpose and complex conjugate). The inner and outer product of $\left|\psi_{0}\right\rangle$ and $\left|\psi_{1}\right\rangle$ is therefore written $\left\langle\psi_{0} \mid \psi_{1}\right\rangle$ and $\left|\psi_{0}\right\rangle\left\langle\psi_{1}\right|$ respectively.

We can decompose a quantum state into a linear combination of basis states,

$$
|\psi\rangle=\sum_{i} c_{i}|i\rangle
$$

for some basis $\{|i\rangle\}$. The coefficients are called amplitudes, and according to the Born rule, their absolute square $\left|c_{i}\right|^{2}$ gives the probability of finding the system in the corresponding basis state $|i\rangle$. Actually, Born's rule says that we should interpret the square of the amplitudes as a probability density (Born 1954), which gives us

$$
\sum_{i}\left|c_{i}\right|^{2}=1
$$

This shows that these states are unit vectors, $\langle\psi \mid \psi\rangle=1$, and go by the name of pure states. In contrast to mixed states that are statistical ensembles of pure states.

As mentioned above, a qubit is a two-dimensional system. The canonical basis for this system we call the computational basis,

$$
\left\{|0\rangle \equiv\left[\begin{array}{l}
1 \\
0
\end{array}\right], \quad|1\rangle \equiv\left[\begin{array}{l}
0 \\
1
\end{array}\right]\right\},
$$


and in this basis, a qubit with state $|\psi\rangle$ can be written as

$$
|\psi\rangle=\left[\begin{array}{l}
c_{0} \\
c_{1}
\end{array}\right]=c_{0}|0\rangle+c_{1}|1\rangle
$$

With $c_{0}$ and $c_{1}$ being complex numbers, a qubit is parametrized by four real parameters, but using the constraint $\left|c_{0}\right|^{2}+\left|c_{1}\right|^{2}=1$ we can reduce them to three. And in polar coordinates Eq. (3.5) can be rewritten as

$$
|\psi\rangle=e^{i \gamma}\left(\cos \left(\frac{\theta}{2}\right)|0\rangle+e^{-i \varphi} \sin \left(\frac{\theta}{2}\right)|1\rangle\right) .
$$

Further, ignoring the global phase, we get the two-parameter expression

$$
|\psi\rangle=\cos \left(\frac{\theta}{2}\right)|0\rangle+e^{-i \varphi} \sin \left(\frac{\theta}{2}\right)|1\rangle,
$$

which has a geometrical representation called the Bloch sphere. The Bloch sphere is shown in Figure 3.1, where pure states reside on the ball's surface, and mixed states reside inside. Antipodal points on the sphere represent orthogonal states, and at the center of the ball is the maximally mixed state. Being a uniform distribution over all states, the maximally mixed state carries no information.

The evolution of a closed quantum system is given by a unitary transformation $\boldsymbol{U}$,

$$
\boldsymbol{U} \boldsymbol{U}^{\dagger}=\boldsymbol{U}^{\dagger} \boldsymbol{U}=\boldsymbol{I}
$$

That is, the system $\left|\psi_{0}\right\rangle$ evolves into $\left|\psi_{1}\right\rangle$ by letting $U$ act on the initial state, $\left|\psi_{1}\right\rangle=U\left|\psi_{0}\right\rangle$. The three most rudimentary transformations of a qubit are the Pauli operators

$$
X=\left[\begin{array}{ll}
0 & 1 \\
1 & 0
\end{array}\right] \quad Y=\left[\begin{array}{cc}
0 & -i \\
i & 0
\end{array}\right] \quad Z=\left[\begin{array}{cc}
1 & 0 \\
0 & -1
\end{array}\right]
$$




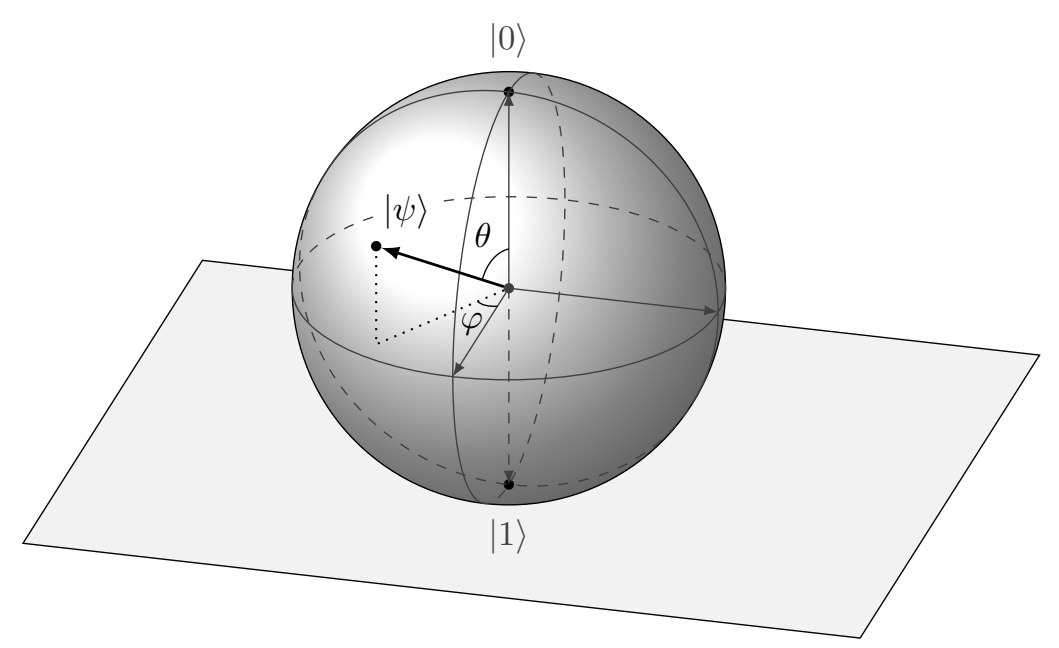

Figure 3.1: The Bloch sphere of a qubit. Pure states reside on the ball's surface, while mixed states live inside. Antipodal points of the sphere correspond to orthogonal states. The illustration shows the computational basis state $|0\rangle$ by convention at the north pole.

which all can be viewed as rotations around the three axes of the Bloch sphere. These rotations leave two pure states invariant, the eigenstates to the operators, and each set of eigenstates form an orthonormal basis for the qubit.

The eigenstates to the $\boldsymbol{Z}$ operator forms the set

$$
\{|0\rangle,|1\rangle\}
$$

which we already know as the computational basis, see Eq. (3.4). The eigenstates to the $\boldsymbol{X}$ operator are

$$
\frac{1}{\sqrt{2}}\left[\begin{array}{l}
1 \\
1
\end{array}\right]=\frac{|0\rangle+|1\rangle}{\sqrt{2}} \equiv|+\rangle
$$

and

$$
\frac{1}{\sqrt{2}}\left[\begin{array}{c}
1 \\
-1
\end{array}\right]=\frac{|0\rangle-|1\rangle}{\sqrt{2}} \equiv|-\rangle,
$$


which forms what we will call the phase-basis, $\{|+\rangle,|-\rangle\}$. And for $\boldsymbol{Y}$ the eigenstates are

$$
\frac{1}{\sqrt{2}}\left[\begin{array}{l}
1 \\
i
\end{array}\right]=\frac{|0\rangle+i|1\rangle}{\sqrt{2}} \equiv|+i\rangle
$$

and

$$
\frac{1}{\sqrt{2}}\left[\begin{array}{c}
1 \\
-i
\end{array}\right]=\frac{|0\rangle-i|1\rangle}{\sqrt{2}} \equiv|-i\rangle,
$$

which form the basis $\{|+i\rangle,|-i\rangle\}$.

We can see the position of these six states on the Bloch sphere in Figure 3.2, and since

$$
\begin{aligned}
& X|0\rangle=|\mathbf{1}\rangle \\
& Y|0\rangle=|\mathbf{1}\rangle \\
& Z|+\rangle=|-\rangle,
\end{aligned}
$$

modulo global phase, these operations can be thought of as $180^{\circ}$ rotations around their respective axis in the Bloch sphere picture. Quantum systems compose under the tensor product, $\otimes$. Consider that we have one system in state $\left|\psi_{1}\right\rangle_{A}$ in a Hilbert space $\mathcal{H}_{A}$ and another system in the state $\left|\psi_{0}\right\rangle_{B}$ in a Hilbert space $\mathcal{H}_{B}$, then the state describing the combined system is

$$
|\psi\rangle_{A B}=\left|\psi_{1}\right\rangle_{A} \otimes\left|\psi_{0}\right\rangle_{B}
$$

in $\mathcal{H}_{A} \otimes \mathcal{H}_{B}$. States that we can write as Eq. (3.16) is called a product state. But but we cannot write all states on this form, and those that we cannot are called entangled states. As an example, the state

$$
\frac{|00\rangle+|11\rangle}{\sqrt{2}}
$$

cannot be written in product form and is therefore an entangled. 


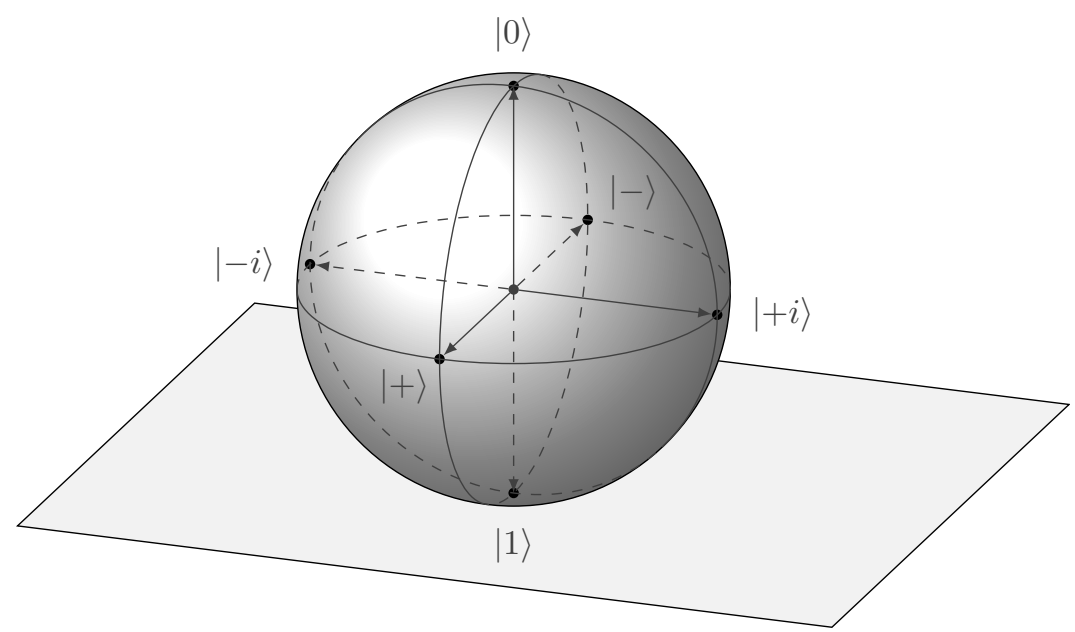

Figure 3.2: Presenting the position of the six states, forming the three ON-bases $\{|0\rangle,|1\rangle\},\{|+\rangle,|-\rangle\}$, and $\{|+i\rangle,|-i\rangle\}$, on the Bloch sphere.

\subsection{Measurement}

Formally, a measurement is described by a set of measurement operators $\left\{M_{i}\right\}$. The measurement operators, together with the state, describe a probability distribution from which we draw a sample $i$, this sample is the outcome of the measurement. The measurement operators obey the completeness relation

$$
\sum_{i} M_{i}^{\dagger} M_{i}=I,
$$

and together with the Born rule, we see that a measurement exhaust the probability space; a measurement always yields an outcome,

$$
\sum_{i}\left\langle\psi\left|M_{i}^{\dagger} M_{i}\right| \psi\right\rangle=1
$$

The terms $\left\langle\psi\left|M_{i}^{\dagger} M_{i}\right| \psi\right\rangle$ represent the probability of $i$ being the outcome. 
According to Lüders' rule (Lüders 2016) a measurement necessarily updates the state to be compatible with the obtained outcome $\boldsymbol{i}$. The state after a measurement is updated according to

$$
\frac{M_{i}|\psi\rangle}{\sqrt{\left\langle\psi\left|M_{i}^{\dagger} M_{i}\right| \psi\right\rangle}} .
$$

The most common measurement is known as a projective measurement, or a von Neumann measurement, and it is represented by a Hermitian operator, $\boldsymbol{A}=\boldsymbol{A}^{\dagger}$, called observable. The operator has a diagonal decomposition

$$
A=\sum_{i} \lambda_{i}\left|e_{i}\right\rangle\left\langle e_{i}\right|
$$

where $\left|e_{i}\right\rangle\left\langle e_{i}\right|$ are orthogonal projection operators constructed from the eigenstates of the operator $\boldsymbol{A}$, and $\boldsymbol{\lambda}_{i}$ the corresponding eigenvalues. In a projective measurement, these projectors are the measurement operators $M_{i}$ and eigenvalues are measurement outcomes.

For projective measurements, the average probability of obtaining $\lambda_{i}$ is $\left|\left\langle\psi \mid e_{i}\right\rangle\right|^{2}$, which is sometimes known as the statistical overlap or the transition probability, since it expresses the probability of $|\psi\rangle$ updating to $\left|e_{i}\right\rangle$ under the measurement.

As an example, consider a projective measurement in the computational basis (measuring the observable $Z$ ) that has the following set of measurement operators $\{|0\rangle\langle 0|| 1\rangle,\langle 1|\}$. If the qubit is in the state $|\psi\rangle=c_{0}|0\rangle+c_{1}|\mathbf{1}\rangle$, the state after measurement becomes $|0\rangle$ with probability $\langle\psi \mid 0\rangle\langle 0 \mid 0\rangle\langle 0 \mid \psi\rangle=|\langle\psi \mid 0\rangle|^{2}=$ $\left|c_{0}\right|^{2}$, and $|\mathbf{1}\rangle$ with probability $\langle\psi \mid \mathbf{1}\rangle\langle\mathbf{1} \mid \mathbf{1}\rangle\langle\mathbf{1} \mid \psi\rangle=|\langle\psi \mid \mathbf{1}\rangle|^{2}=$ $\left|c_{1}\right|^{2}$. 


\subsection{Gates and Notation}

The tensor composition $\left|\psi_{1}\right\rangle \otimes\left|\psi_{0}\right\rangle$ is abbreviated in many ways, sometimes just omitting the tensor notation $\left|\psi_{1}\right\rangle\left|\psi_{0}\right\rangle$, sometimes inside the same ket, $\left|\psi_{1} \psi_{0}\right\rangle$, and sometimes to indicate a separation they are separated by a comma $\left|\psi_{1}, \psi_{0}\right\rangle$.

We can encode binary strings in the computational basis on a composition of qubits. Two examples of encoding bit strings of length three are

$$
|5\rangle \equiv|101\rangle
$$

and

$$
|1\rangle \equiv|001\rangle,
$$

where the rightmost system is the least significant qubit. Note that a string of zeros $\left|0^{n}\right\rangle$ in decimal representation could be denoted $|0\rangle$, and as in Eq. (3.23), the binary encoding of 1, that is $\left|0^{n-1} 1\right\rangle$, could be denoted $|1\rangle$ in the decimal representation. This ambiguity can cause some confusion, but what we mean should be clear from the context.

The most rudimentary operation in information processing is the bit flip operation. In the computational basis, this is done by the Pauli- $\boldsymbol{X}$ operation. (one could also use Pauli- $\boldsymbol{Y}$, but that would introduce a phase shift as well, see Eq. (3.15).) As an example

$$
X \otimes X \otimes I|5\rangle=|3\rangle .
$$

This operation has a diagrammatic representation called a quantum circuit. (see Figure 3.3.) Here the identity operations are

$$
\begin{aligned}
& |1\rangle-|1\rangle \\
& |0\rangle-X-|1\rangle \\
& |1\rangle-X-|0\rangle
\end{aligned}
$$

Figure 3.3: Circuit diagram mapping $|5\rangle$ to $|3\rangle$ 
represented as horizontal wires, and the circuit reads from left to right. That is, states enter the circuit from the left and leave to the right. Also, here the uppermost qubit in the circuit encodes the least significant bit. Observe that some authors have the most significant bit as the topmost bit.

Quantum gates are unitary transformations, and the gate that reverses $\boldsymbol{U}$ is its Hermitian conjugate $\boldsymbol{U}^{\dagger}$. There are quantum gates that relates to all classical reversible gates in Section 2.4. These quantum gates perform the same logical operations over the computational basis, see Figure 3.4.

Here it is important to point out that the circuit notation used for quantum gates is usually interchangeable with that of classical reversible gates. But since we are going to use the notation of classical reversible logic side-by-side with quantum circuitry later on, we use a slightly different notation for quantum gates, see Figure 3.4.

There are also quantum gates that do not have a corresponding classical gate, see Figure 3.5. A first example is the Pauli- $Z$ gate that flips the phase-basis

$$
Z|+\rangle=|-\rangle, \quad Z|-\rangle=|+\rangle
$$

Another common gate is the Hadamard gate, which takes the computational basis into the phase-basis, and vice versa.

$$
\boldsymbol{H}|\mathbf{0}\rangle=|+\rangle, \quad \boldsymbol{H}|\mathbf{1}\rangle=|-\rangle .
$$

There are also gates performing phase rotation. Most common are the $S$ - and the $\boldsymbol{T}$-gate that contributes with a phase shift of $\pi / 2$ and $\pi / 4$ radians, respectively.

$$
\begin{gathered}
S\left(\frac{|0\rangle+|1\rangle}{\sqrt{2}}\right)=\frac{|0\rangle+i|1\rangle}{\sqrt{2}} \\
T\left(\frac{|0\rangle+|1\rangle}{\sqrt{2}}\right)=\frac{|0\rangle+e^{i \frac{\pi}{4}}|1\rangle}{\sqrt{2}} .
\end{gathered}
$$




$$
\begin{aligned}
& -X-\equiv\left[\begin{array}{ll}
0 & 1 \\
1 & 0
\end{array}\right] \\
& \left.-Y-\equiv \quad \begin{array}{rr}
0 & -i \\
i & 0
\end{array}\right] \\
& \underset{-T-}{-i}-\equiv\left[\begin{array}{llll}
1 & 0 & 0 & 0 \\
0 & 1 & 0 & 0 \\
0 & 0 & 0 & 1 \\
0 & 0 & 1 & 0
\end{array}\right] \\
& \underset{\leftarrow}{*} \equiv\left[\begin{array}{llll}
1 & 0 & 0 & 0 \\
0 & 0 & 1 & 0 \\
0 & 1 & 0 & 0 \\
0 & 0 & 0 & 1
\end{array}\right] \\
& \text { ב- } \\
& \underset{*}{*} \equiv\left[\begin{array}{llllllll}
1 & 0 & 0 & 0 & 0 & 0 & 0 & 0 \\
0 & 1 & 0 & 0 & 0 & 0 & 0 & 0 \\
0 & 0 & 1 & 0 & 0 & 0 & 0 & 0 \\
0 & 0 & 0 & 1 & 0 & 0 & 0 & 0 \\
0 & 0 & 0 & 0 & 1 & 0 & 0 & 0 \\
0 & 0 & 0 & 0 & 0 & 0 & 1 & 0 \\
0 & 0 & 0 & 0 & 0 & 1 & 0 & 0 \\
0 & 0 & 0 & 0 & 0 & 0 & 0 & 1
\end{array}\right]
\end{aligned}
$$

Figure 3.4: Quantum gates related to the classical reversible gates, and their unitary matrix representation. 


$$
\begin{array}{ll}
-Z- & \left.\equiv \begin{array}{cc}
1 & 0 \\
0 & -1
\end{array}\right] \\
-H- & \equiv\left[\begin{array}{cc}
1 & 1 \\
1 & -1
\end{array}\right] \\
-S- & \equiv\left[\begin{array}{cc}
1 & 0 \\
0 & i
\end{array}\right] \\
-T- & \equiv\left[\begin{array}{cc}
1 & 0 \\
0 & e^{i \frac{\pi}{4}}
\end{array}\right]
\end{array}
$$

Figure 3.5: Quantum gates that lack classical counterparts, and their unitary matrix representation.

See Figure 3.5 for their circuit and matrix representation.

In the circuit notation, measurement in the computational basis is denoted by a "meter" symbol, see figure Figure 3.6.

A quantum algorithm, or a subroutine, is usually described as a circuit that takes as input a prepared state and outputs a final state. The measurement, together with the output state, specify a probability distribution from which we draw a sample. We then use the information retrieved from this sampling procedure to solve a computational problem.

Let us illustrate this with an example.

Example 3.3.1: Consider the circuit in Figure 3.6. From left to right:

1. Begin by preparing two qubits in the state $|0\rangle|0\rangle$.

2. Put the most significant qubit through a Hadamard gate to produce,

$$
\frac{|00\rangle+|10\rangle}{\sqrt{2}} \text {. }
$$


3. Then apply a CNOT gate with the most significant qubit as the control, and the least significant qubit as target, producing the state

$$
\frac{|00\rangle+|11\rangle}{\sqrt{2}} .
$$

This is an entangled state and one of the four Bell states.

4. At the last step, we perform a measurement in the computational basis, returning that the system is in either state $|00\rangle$ or $|11\rangle$, both with probability $1 / 2$.

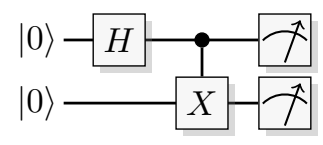

Figure 3.6: Circuit preparing two qubits, both in state $|0\rangle$, transforming it into a Bell state, and then measuring in the computational basis.

Another important transformation that we will use is the WalshHadamard transform, that is, the Fourier transform over the field $\mathbb{Z}_{2}^{n}$. This transformation is obtained by applying a sequence of $\boldsymbol{n}$ Hadamard gates, $\boldsymbol{H}^{\otimes n}$. If applied to an integer state $|\boldsymbol{x}\rangle$ of size $n$, we get

$$
H^{\otimes n}|x\rangle=\frac{1}{\sqrt{2^{n}}} \sum_{y=0}^{2^{n}-1}(-1)^{x \cdot y}|y\rangle
$$

Where $\boldsymbol{x} \cdot \boldsymbol{y}$ denotes the inner product in $\mathbb{Z}_{2}^{n}$. 


\section{Part II}

\section{Summary of the results}





\section{Chapter 4}

\section{Results}

In this section, we give a brief overview of the methods and main results of the publications in Part III.

\subsubsection{Publication A}

In Publication A, we work with Spekkens' model (Spekkens 2007). We devised an efficient representation of this model, and we extended the model to enable investigations of algorithms similar to quantum algorithms. The extension we call Quantum Simulation Logic (QSL).

Spekkens' model can reproduce many quantum phenomena, and it has a clear graphical representation ${ }^{1}$. The state space is partitioned into a grid, and probability distributions over the grid represent states. Permutations of the partitions represent transformations, and there is an explicit measurement procedure. This representation is clear, but it is not efficient; working with large systems quickly becomes intractable. So we encoded the state space on bit-strings. Transformations are then given by reversible logical gates acting on these bit-strings, and the

\footnotetext{
${ }^{1}$ Later also an analytical representation (Spekkens 2016).
} 
measurement procedure consists of reading one bit value and randomizing another.

An elementary system has two degrees of freedom, and we encode these in two bits. We call these bits the computational-bit and the phase-bit. The effect of some gates that correspond to oneand two-qubit quantum gates are explicit in this representation. Of particular interest is the effect that corresponds to what is usually called phase kickback.

To compare with circuits used in quantum algorithms, we also needed a gate corresponding to the Toffoli gate. Here we decided to apply a classical Toffoli gate over the computational bits and no effect over the phase bits. That is, the Toffoli gate does not have a phase kickback effect in Publication A. This gate, together with the new representation of Spekkens' model, is what we call QSL.

We showed that there exist QSL oracles for both the DEUTSCHJozsA and Simon's algorithms, and relative to these, we can solve the corresponding computational problems with the same number of queries as in the quantum case.

QSL carries only a constant overhead compared with quantum circuits. QSL requires two classical bits for each simulated qubit, and it requires at most two classical reversible gates for each simulated quantum gate. That is, for a quantum circuit of size $k$, the QSL simulation will be a classical reversible circuit of size (at most) $2 k$.

It is important to stress that QSL is an approximation far from simulating universal quantum computation. What is interesting is that such crude approximation contains oracles enabling the mentioned algorithms.

In the Deutsch-JozSA and Simon's algorithm, we assume access to a quantum oracle. Comparing with access to an oracle that only computes the function, we get an exponential speedup. Instead, comparing access to a quantum oracle with access to a 
QSL oracle, we get no speedup at all. Also considering the fact that QSL is a classical circuit with a constant overhead, we have efficient classical simulations for both the DeUTSCH-Jozsa and Simon's algorithm.

\subsubsection{Publication B}

Publication B is a substantial extension of Publication A. Here we modify QSL by altering the Toffoli gate by adding an effect on the phase-bits. This effect is constructed to approximate the phase kickback in a quantum Toffoli gate. The modification enables QSL to better approximate quantum circuits, but it is still far from simulating universal quantum computation. For instance, QSL cannot reproduce non-local correlations or contextual relations. Furthermore, the modified Toffoli gate only uses three classical reversible gates, so even with this modification, QSL only carries a constant overhead compared with quantum circuits. Observe that we still call this model QSL.

We verify that the results in Publication A still hold under this modification. That is, there exist oracles in QSL that allow us to solve the Deutsch-JozSA and Simon's problem with the same number of queries as when given access to a quantum oracle. It also works for both the promise and decision version of the DEuTsch-Jozsa problem, and for the exact quantum algorithm for Simon's problem.

There are also oracles for the BERNSTEIN-VAZIRANI, and GROvER's problem. For BERnStEIn-VAZirani the number of queries is the same as in the quantum case, but for Grover's there is only a logarithmic speedup (compared to a quadratic speedup in the quantum case). QSL also gives good approximations for some explicit construction of SHOR's and GROVER's algorithms for small problem instances. For more examples that compare QSL with quantum theory, we direct the reader to Publication B.

We can again conclude that, relative to the QSL oracles, there 
are efficient classical algorithms for the problems solved by the Bernstein-VAzirani, Deutsch-Jozsa, and Simon's algorithm. In fact, the query complexity is exactly the same. It follows that, there is also a speedup in QSL (compared with access to a regular oracle). Further, the speedup is the same as the proposed quantum speedup, and there is no doubt that the property enabling the speedup in QSL is the ability to store, process, and retrieve information in an additional degree of freedom.

In general, we strongly question whether comparing bounds from classical query complexity with those from quantum query complexity can provide conclusive evidence for a quantum advantage.

\subsubsection{Publication C}

In this publication, we part from QSL but continue pursuing the idea that the enabling property of quantum speedup is the availability of an additional degree of freedom.

Most of our focus is on what we call the phase degree of freedom, which is the eigenbasis of Pauli $Z$ operators, $\{|+\rangle,|-\rangle\}^{n}$. This basis is unbiased with the computational basis, meaning that a projective measurement onto one basis does not give us any information about the other. Together with the fact that we can vary the phase of a quantum state independently of the amplitude, we relate the computational basis and the phase basis to two different degrees of freedom. Instead of describing gates and circuits in the computational basis, we describe them in the phase basis. We then use this description to analyze the effect of quantum circuits in the phase degree of freedom.

We start with FourIER SAMPLING, which is an important procedure used in many quantum algorithms. FourIER SAMPLING includes a reversible function that is encoded into the computational basis of a unitary transformation. The function reads data from the input register and writes the result to the output register. 
In the phase basis, it is the other way around; the computation reads data from the output register and writes the result to the input register. In FOURIER SAMPLING we retrieve the result by measuring the input register, which fits well with its description in the phase degree of freedom.

Both the Deutch-Jozsa and the Bernstein-Vazirani algorithms are direct applications of Fourier SAMPLING. Fourier SAMPLING is also part of GROVER's algorithm, and both SIMON'S and SHOR's algorithm can be seen as alterations of FourIER SAMPLING. We can adapt our method to all of these quantum algorithms, but to get a more general result we show that it applies to the HADAMARD TEST, which can be used to solve a promiseBQP-complete problem.

For a problem to be promiseBQP-complete (usually just called BQP-complete) it needs to be included in BQP and have the property that any other problem in BQP can be efficiently reduced to it. In this way, it relates to all problems that can be efficiently solved with a quantum computer. Observe that all problems in BQP now can be reduced and solved using the Hadamard Test.

The Hadamard Test can be described as using one function map in the computational basis in the forward direction, and another function map in the phase degree of freedom in the reverse direction. Thus we draw the conclusion that all quantum algorithms that solve a problem in $\mathbf{B Q P}$ can be described using one function map in the computational basis in the forward direction, and another function map in the phase degree of freedom in the reverse direction. (At least after reduction to the promiseBQPcomplete problem.) The conditions on relative phases specify the function in the phase degree of freedom. In turn, these conditions are specified in either the problem formulation or by the quantum algorithm that solves the problem.

We conclude that the property enabling the potential quantum speedup is the ability to store, process, and retrieve information 
in an additional degree of freedom. How the computation is allowed to process information in the additional degree of freedom is specified by the quantum algorithm.

\subsubsection{Publication D}

Looking at quantum error correction, we saw that a quantum error correcting code, called the five qubit code, also worked in Spekkens' model. This code is what is called a stabilizer code, and our hypothesis was that stabilizer codes could be identified with classical error correcting codes over two degrees of freedom.

Stabilizer codes are usually described with the stabilizer representation, and we aimed to construct a similar representation for Spekkens' model. At this stage in our research we realized that the stabilizer representation can be interpreted as a propositional logic. The construction of this propositional logic, which we call conjugate logic, is the subject of Publication D.

Phase space logic contains three truth values, true, false, and uncertain. Strings of Pauli operators represent propositions, and these encode knowledge about a system. More precisely, they are statements about future measurement outcomes of the underlying system.

The system can be subject to a transformation, and in conjugate logic, transformations are handled by a corresponding relabeling of the propositions. These re-labeling operations we simply call transformations.

The construction is not fixed, that is, there are choices that we can make when defining the transformations. Of particular interest are the transformations that correspond to the Hadamard and $C Z$ transformations. One choice make conjugate logic describe Spekkens' model while making the other choice makes it describe the stabilizer subtheory ${ }^{2}$ of quantum mechanics.

\footnotetext{
${ }^{2}$ Quantum theory restricted to Clifford group transformations and Pauil
} 
Spekkens model is non-contextual. That is, measurements do not depend on their context (set of simultaneously measurable observables), and we can pre-assign values to all observables. This is not true for the stabilizer subtheory, which is a contextual model. Therefore, in conjugate logic, we can see how contextuality emerges from the choices made for the Hadamard and $C Z$ transformations.

We hope to extend this also to include QSL, and that this tool can be used to investigate QSL and similar existing models further. Perhaps it could even inspire to the creation of new models.

\subsection{Conclusions}

When we compare between quantum and classical oracles, we compare access to $\boldsymbol{U}_{\boldsymbol{f}}|\boldsymbol{x}, \boldsymbol{y}\rangle=|\boldsymbol{x}, \boldsymbol{y} \oplus \boldsymbol{f}(\boldsymbol{x})\rangle$ with access to the classical reversible function $f:(x, y) \mapsto(x, y \oplus f(x))$, that is encoded in the quantum oracle. But it should now be clear that access to a unitary map encoding a function $f$ (along with further conditions) also includes access to another function. Let us call this function $g$. Specifically, it is the conditions on relative phases that specify this function. The conditions on relative phases are made visible by letting $\boldsymbol{U}_{f}$ act on a general quantum state,

$$
U_{f} \sum_{x, y} c_{x, y}|x, y\rangle=\sum_{x, y} c_{x, y}|x, y \oplus f(x)\rangle .
$$

From this we see that $\boldsymbol{U}_{f}$ preserves the coefficients $\boldsymbol{c}_{x, y}$, and therefore also the relative phases.

One particular oracle problem where it is especially clear that the unitary gives us access to two different functions is the BERNSTEINVazirani problem (Bernstein and Vazirani 1997). Also here, the quantum algorithms that solve this problem is the FourIER SAMPLING procedure. In the BernsteIn-VAZIRANI problem, we are group measurement. 
given access to a Boolean function promised to be on the form $f_{B V}(x)=x \cdot s$, where $s$ is a secret string. Informally, the problem is to retrieve $s$. With access only to the Boolean function, this would require a number of queries linear in the number of bits needed to specify the input. A quantum computer with access to the standard phase-preserving unitary map corresponding to that function only requires a single query.

Using the Walsh-Hadamard-transformed unitary

$$
\boldsymbol{H}^{\otimes n+1} U_{f_{B V}} \boldsymbol{H}^{\otimes n+1}|\boldsymbol{x}, \boldsymbol{y}\rangle=|\boldsymbol{x}+\boldsymbol{y} \boldsymbol{s}, \boldsymbol{y}\rangle
$$

we find that the quantum oracle gives us access to the function $g_{B V}: \mathbb{Z}_{2}^{n} \times \mathbb{Z}_{2} \rightarrow \mathbb{Z}_{2}^{n} \times \mathbb{Z}_{2},(x, y) \mapsto(x+y s, y)$. By querying this function with $y=1$ and $x=0^{n}$ we directly retrieve the secret string $s$.

The question is now why we should compare a quantum oracle with a classical oracle computing $f$, and not with a classical oracle computing $\boldsymbol{g}$, or indeed even a classical oracle computing the pair $(f, g)$. The function $f$ is available in the quantum oracle, but so is $g$, and it is $g$ that we access to solve the computational problem. Further, the condition that $f$ is encoded in a unitary map is a strong condition, but it is not enough. There are many further requirements on the unitary before we can access $g$. These requirements include, among others, the conditions on relative phases. See Publication $\mathrm{C}$ for details.

In fact, if we only require the quantum operation to encode $g$ in some basis, we do not need any additional requirements on the relative phases. The presence of $g$ in the output state is enough. The quantum operation does not even need to be unitary. It can be completely phase mixing, as long as the function $g$ can be accessed in the output. From this we conclude that the quantum operation should be compared with access to $g$, or access to $f$, when the experimenter so chooses.

Further, comparing with classical query complexity bounds there is a speedup, but to relate these bounds to the computational 
power of a physical process we need to make at least one assumption. Deutsch and Jozsa 1992 writes.

"Let $\boldsymbol{O}_{f}$ be a device that computes a function $f: \mathbb{Z}_{m} \rightarrow$ $\mathbb{Z}_{n}$. Given an input $\boldsymbol{i}, \boldsymbol{O}_{f}$ will, after some time, output the value $f(i)$. In general terms the class of computational task which we shall be considering involves being given $\boldsymbol{O}_{f}$ and then using it to determine some property $G[f]$ (that is, some function $G$ of the sequence $f(0), f(1), \ldots, f(m-1)$ in the least possible time. In the analysis of this type of task, it is often an excellent approximation that the internal workings of $\boldsymbol{O}_{f}$ are inaccessible, in which case $\boldsymbol{O}_{f}$ is known as an oracle. The approximation would be nearly exact if $\boldsymbol{O}_{f}$ were a new type of physical object with an unknown law of motion. If $\boldsymbol{O}_{\boldsymbol{f}}$ were simply a program for evaluating $\boldsymbol{f}$ on our computer, making the approximation is tantamount to assuming that there is no faster method of obtaining $G[f]$ from the program $O_{f}$ (e.g. by a textual analysis) than actually executing $O_{f}$ to obtain sufficiently many values $f(i)$ to determine $G[f]$."

For the circuit model this assumption can be restated as: there is no faster method of obtaining a property $G[f]$ than to run the circuit computing $f$ to obtain sufficiently many values $f(i)$ to determine the property $G[f]$.

From our perspective, quantum computation is a counterexample to this assumption. A quantum circuit can be seen as a circuit carefully constructed to leak information in a different degree of freedom. Sometimes this information allows us to draw conclusions about a property of the function, and indeed, we can choose to either retrieve this information or to calculate values of $f$.

From what we have presented in this chapter, supported in detail by the included publications, we conclude that. 
- Comparing bounds from classical query complexity with those from quantum query complexity does not provide conclusive evidence for a quantum advantage.

- The property enabling the potential quantum speedup (problems in BQP) is the ability to store, process, and retrieve information in an additional degree of freedom. How the computation process information in the additional degree of freedom is specified by the quantum algorithm.

\subsection{Future Work}

We have given many examples of quantum phenomena from the point of view of different degrees of freedom, and there are many applications in quantum technology that one could look into further. For instance, one could consider quantum error correcting codes. It is possible that, particularly stabilizer codes, can be identified with corresponding classical codes over several degrees of freedom.

In QSL there is room for improvement. We simulate one qubit with two classical bits and a small number of classical gates. Increasing the number of bits and gates could give us a better approximation. A natural progression would be to consider the eight-state model (three bits) (Wallman and Bartlett 2012; Blasiak 2013) and adding a construction for the Toffoli gate. The eight-state model has been proposed as a contextual model (Blasiak 2015), and indeed, the Hadamard transformation in this model should correspond to the transformation in Publication D for which contextuality emerges.

On another topic, quantum computation poses a serious threat to cryptography. Primarily because of Shor's algorithm, which can be used to attack asymmetric protocols that build their security around integer factorization or the discrete logarithm problem. There are alternative protocols, but these have not been investi- 
gated to the same extent. To better understand the limitations of quantum computation will be an important step in adding confidence in these alternative protocols.

A quantum circuit can be interpreted as a circuit that is carefully constructed to leak information in a different degree of freedom. In cryptography this is known as a side-channel. A side-channel attack usually exploits vulnerabilities that appear when implementing a protocol in a physical system. Therefore, it is seen as a vulnerability of the implementation rather than a vulnerability in the protocol. It is not only Shor's algorithm that is seen as a threat in cryptography. There are other protocols that have been found vulnerable to quantum attacks (Kuwakado and Morii 2010; Kuwakado and Morii 2012; Kaplan et al. 2016). We conjecture that these are side-channel attacks, but in general this needs further investigation. 



\section{Bibliography}

Aaronson, Scott and Daniel Gottesman (2004). "Improved Simulation of Stabilizer Circuits”. In: Physical Review A 70. DOI: 10.1103/PhysRevA.70.052328.

Arora, Sanjeev and Boaz Barak (2009). Computational Complexity: A Modern Approach. 1st. New York, NY, USA: Cambridge University Press.

ISBN: 978-0-521-42426-4.

Arute, Frank et al. (2019). "Quantum Supremacy Using a Programmable Superconducting Processor”. In: Nature 574, pp. 505510.

DOI: $10.1038 / \mathrm{s} 41586-019-1666-5$.

Baumgratz, T., M. Cramer, and M. B. Plenio (2014). "Quantifying Coherence”. In: Physical Review Letters 113, p. 140401. DOI: 10.1103/PhysRevLett.113.140401.

Bell, John S. (1964). "On the Einstein Podolsky Rosen Paradox". In: Physics (Long Island City, N. Y.) 1, pp. 195-200.

Bennett, Charles H. (1973). "Logical Reversibility of Computation”. In: IBM J. Res. Dev. 17, pp. 525-532.

DOI: $10.1147 / \mathrm{rd} .176 .0525$. 
Bennett, Charles H. and David P. DiVincenzo (2000). "Quantum Information and Computation”. In: Nature 404, pp. 247-255. DOI: $10.1038 / 35005001$.

Bermejo-Vega, Juan et al. (2017). "Contextuality as a Resource for Models of Quantum Computation with Qubits”. In: Phys. Rev. Lett. 119, p. 120505. DoI: 10.1103/PhysRevLett.119.120505.

Bernstein, Ethan and Umesh Vazirani (1993). "Quantum Complexity Theory”. In: Proceedings of the Twenty-Fifth Annual ACM Symposium on Theory of Computing. STOC '93. New York, NY, USA: ACM, pp. 11-20. DOI: $10.1145 / 167088.167097$.

- (1997). “Quantum Complexity Theory”. In: SIAM Journal on Computing 26, pp. 1411-1473. DOI: $10.1137 / \mathrm{S} 0097539796300921$.

Blasiak, Pawel (2013). "Quantum Cube: A Toy Model of a Qubit”. In: Phys. Lett. A 377, pp. 847-850. DoI: $10.1016 / j$.physleta.2013.01.045.

- (2015). "Classical Systems Can Be Contextual Too: Analogue of the Mermin-Peres Square”. In: Annals of Physics 353, pp. 326-339. DOI: $10.1016 /$ j.aop. 2014.10.016.

Born, Max (1954). "The Statistical Interpretation of Quantum Mechanics”. In: Nobel Lecture 11, pp. 257-267.

Bravyi, Sergey, David Gosset, and Robert König (2018). "Quantum Advantage with Shallow Circuits". In: Science 362, pp. 308311.

DOI: $10.1126 /$ science.aar3106.

Briegel, H. J. et al. (2009). "Measurement-Based Quantum Computation”. In: Nature Phys 5, pp. 19-26.

DOI: $10.1038 /$ nphys 1157. 
Deutsch, David (1985). "Quantum Theory, the Church-Turing Principle and the Universal Quantum Computer”. In: Proc. Roy. Soc. London A 400, pp. 97-117.

DOI: $10.1098 /$ rspa. 1985.0070.

Deutsch, David and Richard Jozsa (1992). "Rapid Solution of Problems by Quantum Computation”. In: Proceedings of the Royal Society of London A: Mathematical, Physical and Engineering Sciences 439, pp. 553-558.

DOI: $10.1098 /$ rspa. 1992.0167.

Einstein, A., B. Podolsky, and N. Rosen (1935). "Can QuantumMechanical Description of Physical Reality be Considered Complete?" In: Phys. Rev. 47, pp. 777-780.

DoI: $10.1103 /$ PhysRev .47.777.

Feynman, Richard P. (1982). "Simulating physics with computers”. In: Int. J. Theor. Phys. 21, pp. 467-488.

DOI: $10.1007 / \mathrm{BF} 02650179$.

- (1985). “Quantum Mechanical Computers”. In: Optics News 11, p. 11.

DOI: $10.1364 / 0 N .11 .2 .000011$.

- (1986). “Quantum Mechanical Computers”. In: Foundations of physics 16 , pp. 507-531.

Fredkin, Edward and Tommaso Toffoli (1982). "Conservative Logic”. In: International Journal of Theoretical Physics 21, pp. 219-253.

DOI: $10.1007 / \mathrm{BF} 01857727$.

Gottesman, Daniel (1998). "The Heisenberg Representation of Quantum Computers”. In: arXiv: quant-ph/9807006.

Hillery, Mark (2016). "Coherence as a Resource in Decision Problems: The Deutsch-Jozsa Algorithm and a Variation". In: Physical Review A 93, p. 012111.

DoI: $10.1103 /$ PhysRevA.93.012111. 
Howard, Mark et al. (2014). "Contextuality Supplies the 'Magic' for Quantum Computation”. In: Nature.

DOI: $10.1038 /$ nature 13460 .

Johansson, Niklas, Felix Huber, and Jan-Åke Larsson (To appear). “Conjugate Logic”. In: The Quantum-Like Revolution A Festschrift for Andrei Khrennikov. Ed. by A. Plotnitsky and E. Haven. Springer.

Johansson, Niklas and Jan-Åke Larsson (2017). "Efficient Classical Simulation of the Deutsch-Jozsa and Simon's Algorithms". In: Quantum Inf Process 16, p. 233. DOI: $10.1007 / \mathrm{s} 11128-017-1679-7$.

- (2019). "Quantum Simulation Logic, Oracles, and the Quantum Advantage”. In: Entropy 21, p. 800.

DOI: $10.3390 / \mathrm{e} 21080800$.

- (2021). "Quantum computation and the additional degrees of freedom”. In: Preprint.

Jozsa, R. and N. Linden (2003). "On the Role of Entanglement in Quantum-Computational Speed-Up”. In: Proceedings of the Royal Society A: Mathematical, Physical and Engineering Sciences 459, pp. 2011-2032.

DOI: $10.1098 /$ rspa. 2002.1097.

Kaplan, Marc et al. (2016). "Breaking Symmetric Cryptosystems Using Quantum Period Finding”. In: Advances in Cryptology - CRYPTO 2016. Lecture Notes in Computer Science. Springer, Berlin, Heidelberg, pp. 207-237.

DOI: $10.1007 / 978-3-662-53008-5 \_8$.

Kochen, Simon and E. P. Specker (1967). "The Problem of Hidden Variables in Quantum Mechanics". In: Journal of Mathematics and Mechanics 17, pp. 59-87.

DOI: 10 .1007/978-94-010-1795-4_17.

Kuwakado, H. and M. Morii (2010). "Quantum Distinguisher between the 3-Round Feistel Cipher and the Random Permutation”. In: 2010 IEEE International Symposium on In- 
formation Theory, pp. 2682-2685.

DOI: $10.1109 /$ ISIT . 2010.5513654.

- (2012). "Security on the Quantum-Type Even-Mansour Cipher”. In: 2012 International Symposium on Information Theory and Its Applications, pp. 312-316.

ISBN: 978-4-88552-267-3.

Landauer, Rolf (1961). "Irreversibility and Heat Generation in the Computing Process". In: IBM Journal of Research and Development 5, pp. 183-191.

DOI: $10.1147 / \mathrm{rd} .53 .0183$.

Larsson, Jan-Åke (2012). "A contextual extension of Spekkens' toy model". In: AIP Conference Proceedings. Foundations of Probability and Physics - 6. Vol. 1424. AIP Publishing, pp. 211-220.

DOI: $10.1063 / 1.3688973$.

Lüders, Gerhart (2016). "Concerning the State-change Due to the Measurement Process”. In: Annalen der Physik 15. English translation and discussion by K. A. Kirkpatrick, pp. 663-670. DOI: 10.1002 /andp. 200610207.

Nielsen, Michael A. and Isaac L. Chuang (2010). Quantum Computation and Quantum Information. 10th anniversary ed. Cambridge ; New York: Cambridge University Press. 676 pp. ISBN: 978-1-107-00217-3.

Shor, Peter W. (1994). "Algorithms for Quantum Computation: Discrete Logarithms and Factoring". In: Proceedings 35th Annual Symposium on Foundations of Computer Science. Proceedings 35th Annual Symposium on Foundations of Computer Science, pp. 124-134.

DOI: $10.1109 /$ SFCS . 1994. 365700.

Spekkens, Robert W. (2007). "Evidence for the Epistemic View of Quantum States: A Toy Theory”. In: Phys.Rev.A 75, p. 032110. DOI: $10.1103 /$ PhysRevA.75.032110. 
Spekkens, Robert W. (2016). "Quasi-Quantization: Classical Statistical Theories with an Epistemic Restriction". In: Quantum Theory: Informational Foundations and Foils. Ed. by Giulio Chiribella and Robert W. Spekkens. Fundamental Theories of Physics. Springer Netherlands, pp. 83-135. DOI: 10.1007/978-94-017-7303-4_4.

Toffoli, Tommaso (1980). "Reversible Computing". In: International Colloquium on Automata, Languages, and Programming. Springer, pp. 632-644.

Veitch, Victor et al. (2012). "Negative Quasi-Probability as a Resource for Quantum Computation”. In: New J. Phys. 14, p. 113011.

DOI: $10.1088 / 1367-2630 / 14 / 11 / 113011$.

Wallman, Joel J. and Stephen D. Bartlett (2012). "Non-Negative Subtheories and Quasiprobability Representations of Qubits". In: Phys. Rev. $A$ 85, p. 062121.

DOI: 10.1103/PhysRevA.85.062121. 


\section{Part III}

\section{Publications}





\section{Papers}

The papers associated with this thesis have been removed for copyright reasons. For more details about these see:

https://doi.org/10.3384/9789179291242 



\section{FACULTY OF SCIENCE AND ENGINEERING}

Linköping Studies in Science and Technology, Dissertation No. 2191, 2021 Department of Electrical Engineering

Linköping University

SE-581 83 Linköping, Sweden

wWw.liu.se
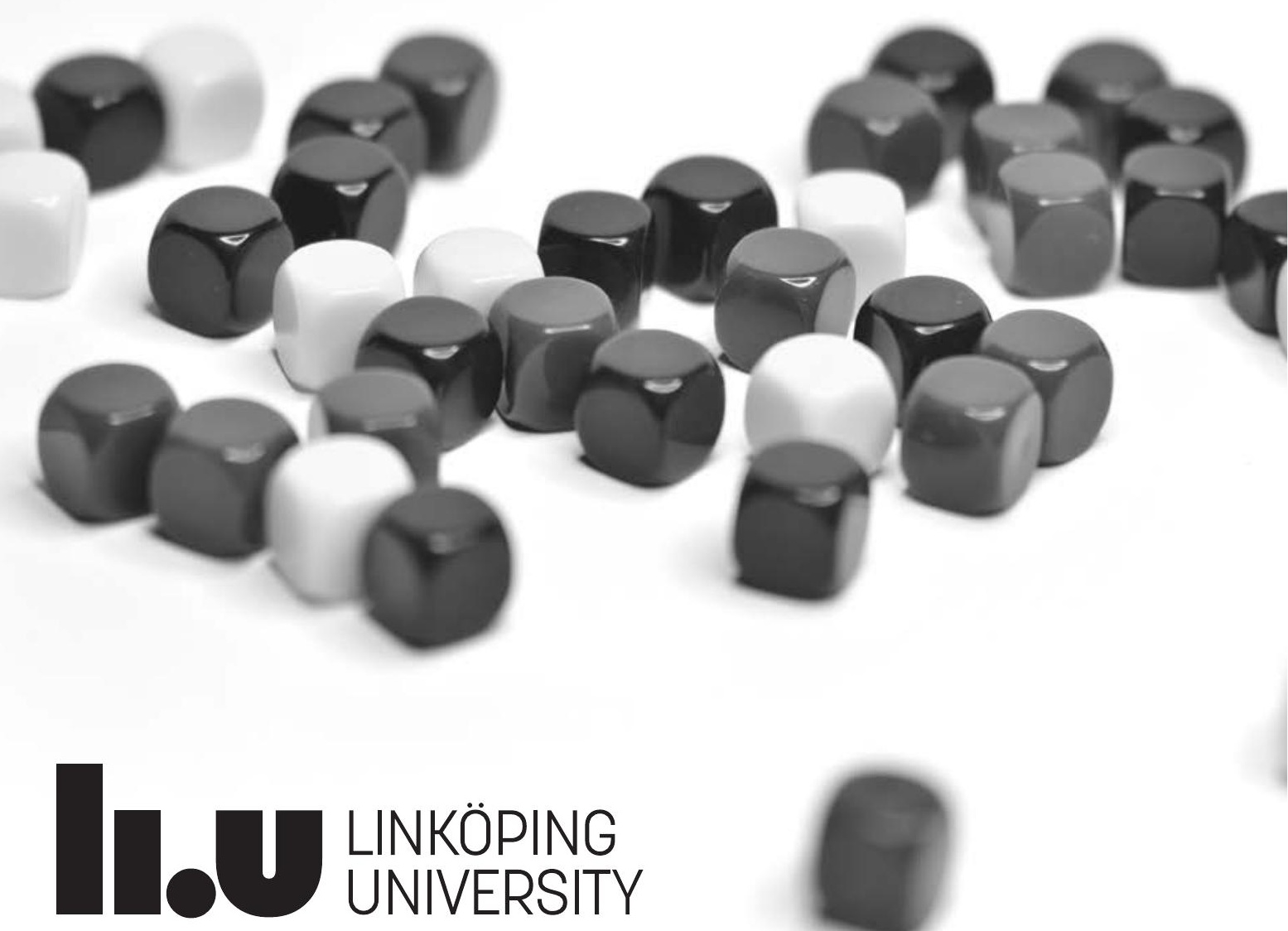